\title{
Loss of the cell polarity determinant human Discs-large is a novel molecular marker of nodal involvement and poor prognosis in endometrial cancer
}

\author{
Takeru Sugihara ${ }^{1}$, Shunsuke Nakagawa ${ }^{*}{ }^{1}$, Yuko Sasajima $^{2}$, Takayuki Ichinose ${ }^{1}$, Haruko Hiraike ${ }^{1}$, \\ Fukuo Kondo ${ }^{2}$, Hiroshi Uozaki ${ }^{2}$, Toshio Fukusato ${ }^{2}$ and Takuya Ayabe ${ }^{1}$ \\ ${ }^{1}$ Department of Obstetrics and Gynecology, Graduate School of Medicine, Teikyo University, Tokyo, Japan and ${ }^{2}$ Department of \\ Pathology, Graduate School of Medicine, Teikyo University, Tokyo, Japan
}

Background: Recent Drosophila studies showed that Discs-large (Dlg) is critical for regulation of cell polarity and tissue architecture. We investigated the possibility that loss of the human homologue of Drosophila Dlg (DLG1) is involved in endometrial carcinogenesis.

Methods: We analysed DLG1 expression in 160 endometrial cancers by immunohistochemical staining. Its expression was confirmed by quantitative real-time PCR (RT-PCR). We investigated the roles of DLG1 in growth and invasion by knockdown experiment in endometrial cancer cell lines.

Results: Human DLG1 localises at cellular membrane in normal endometrial tissues. Loss of DLG1 was observed in 37 cases (23.1\%). Loss of DLG1 was observed in patients with advanced stage and high-grade histology. It was also observed in patients with nodal metastasis, deep myometrial invasion, and negative oestrogen and progesterone receptors. Patients with loss of DLG1 showed poorer overall survival $(P=0.0019)$. Immunohistochemistry data correlated with RT-PCR data. Knockdown of Dlg1 in endometrial cancer cells resulted in accelerated tumour migration and invasion in vitro.

Conclusions: Tissue polarity disturbance because of loss of DLG1 was shown to confer more aggressive characteristics to endometrial cancer cells. Our study revealed that DLG1 expression is a novel molecular biomarker of nodal metastasis, high-grade histology, and poor prognosis in endometrial cancer.

Endometrial cancer is the most common gynaecological malignancy in developed countries with an estimated worldwide incidence of 287000 cases and 74000 deaths in 2008 (Ferlay et al, 2010). Endometrial cancer is classified into two groups, type 1 and type 2, based on pathological histology, as well as molecular pathogenesis and clinical profiles (Bokhman, 1983). Type 1 endometrial cancer is oestrogen dependent, has low-grade endometrioid histology, and arises in a background of endometrial hyperplasia, its precursor lesion (Doll et al, 2008). Type 1 endometrial cancer usually occurs in pre- and peri-menopausal women and is strongly linked to obesity (Doll et al, 2008). Type 2 endometrial cancer is oestrogen independent and has high-grade histology, that is, high-grade endometrioid, papillary serous, and clear cells. It usually occurs in nonobese and post-menopausal elderly women (Mendivil et al, 2009). Although type 2 endometrial cancer accounts for only $10 \%$ of the incidence of endometrial cancer, it is responsible for $\sim 50 \%$ of disease recurrence (Bansal et al, 2009).

*Correspondence: Dr S Nakagawa; E-mail: nakagawas-tky@umin.ac.jp

Received 30 May 2015; revised 4 October 2015; accepted 13 January 2016; published online 22 March 2016

(c) 2016 Cancer Research UK. All rights reserved 0007-0920/16 
The 5-year survival rate for endometrial cancer is $96 \%$ if the cancer is diagnosed at a local stage, but it decreases to $17 \%$ if it is diagnosed at an advanced stage (Jemal et al, 2010). Deep myometrial invasion, nodal involvement, and distant metastasis worsen the prognosis of endometrial cancer (Barrena Medel et al, 2011). The identification of biomarkers that predict deep invasion and metastasis in endometrial cancer is thus needed. A recent study showed that the genomic features of endometrial cancer permit a reclassification of endometrial cancer patients for their prognosis (Cancer Genome Atlas Research Network, et al, 2013).

Loss of cellular polarity is a hallmark of cancer (Hanahan and Weinberg, 2011). Reduced cellular adhesion leads to tissue architecture disruption and invasion into surrounding tissues (Hanahan and Weinberg, 2011). Human epithelial tumours lose polarity during the progression of carcinogenesis, but it remains unclear whether polarity loss might causally contribute to cancer development and give metastatic potential. In Drosophila, mutations in three neoplastic tumour suppressor genes, scribble, discs large, and lethal giant larvae, disrupt epithelial tissue polarity, induce extensive overproliferation, and generate invasive and metastatic ability (Bilder, 2004).

Here, we investigated whether the human homologue of the Drosophila tumour suppressor Discs-large (DLG1) is involved in endometrial carcinogenesis. Human DLG1 belongs to the membrane-associated guanylate kinase family and shares protein structural domains, including $3 \mathrm{PDZ}$ domains, an $\mathrm{SH} 3$ domain, and a guanylate kinase-like domain, with its Drosophila homologue (Ivanova et al, 2011). We analysed whether loss of the cellular polarity protein DLG1 is involved in tumour invasion, nodal metastasis, and prognosis for patients with endometrial cancer. We also investigated whether DLG1 has a critical role in migration and invasion using endometrial cancer-derived cell lines.

\section{MATERIALS AND METHODS}

Patients and tumour samples. We analysed DLG1 expression in 160 cases of endometrial cancer who underwent surgery at the university hospital from January 2003 to December 2012 using immunohistochemical staining and compared DLG1 expression with clinical data. All patients provided informed consent to participate in this study. Haematoxylin and eosin (H\&E)-stained slides of these cases were reviewed. We analysed DLG1 expression in 29 endometrial cancer tissues obtained from patients who underwent surgery at the university hospital from September 2011 and in 8 cell lines derived from these tissues by quantitative realtime PCR (RT-PCR). The clinical and pathological characteristics of the cases were obtained from their clinical charts.

Total RNA isolation and cDNA synthesis. Total RNA was extracted using an RNeasy Mini Kit (QIAGEN, Hamburg, Germany) according to the manufacturer's instructions. Complementary DNA (cDNA) was obtained using a SuperScript VILO cDNA Synthesis Kit (Invitrogen, Carlsbad, CA, USA) according to the manufacturer's protocols after total RNA was extracted.

Quantitative RT-PCR of DLG1. The targeted region of DLG1 cDNA was amplified by RT-PCR using cDNA obtained from endometrial cancer and normal endometrial tissues. The PCR conditions were $95^{\circ} \mathrm{C}$ for $10 \mathrm{~s}$ and $60^{\circ} \mathrm{C}$ for $30 \mathrm{~s}$ for 45 cycles. The PCR was performed on a LightCycler 480 (Roche Applied Science, Tokyo, Japan) using the following primer sequences: forward primer: 5'-CCTTCTACAAGAACAAGGACCAG-3'; reverse primer: 5'-GCTGGCATTAGAAGTTACATGCT-3'. A PCR universal probe was used (no. 15, GAGCAGGA, catalogue number 04685148001; Roche Applied Science). The PCR reaction amplified a 74-bp product of human Discs-large (hDlg) cDNA (5'-CCCC TTCTACAAGAACAAGGACCAGAGTGAGCAGGAAACAAGT
GATGCTGACCAGCATGTAACTTCTAATGCC- $3^{\prime}$ ). The targeted region was in exon 19. In addition, the search also included an intron spanning assay. The length of introns spanned by the PCR was $7325 \mathrm{bp}$. $\beta$-Actin expression was selected as the internal control. The Universal Probe Library Human ACTB Gene Assay was used to detect $\beta$-actin expression (catalogue number 05046165001).

Immunohistochemistry (IHC). Paraffin sections, $4 \mu \mathrm{m}$ thick, from representative tumour blocks were screened for DLG1 protein expression using Envision FLEX (DAKO, Glostrup, Denmark). After deparaffinisation and warm bath processing, the sections were activated with citric acid buffer and endogenous peroxidase activity was removed by $3 \% \mathrm{H}_{2} \mathrm{O}_{2}$. After blocking, the sections were incubated for $30 \mathrm{~min}$ with a primary mouse monoclonal anti-DLG1 antibody (2D11, sc-9961, dilution: 1:40; Santa Cruz, Dallas, TX, USA), primary monoclonal mouse anti-Ecadherin antibody (36B5, NCL-E-Cad, dilution: 1:50; Novocastra, Newcastle, UK), primary monoclonal mouse anti-p53 antibody (DO-7, M7001, dilution: 1:50; DAKO), primary monoclonal mouse anti-oestrogen receptor antibody (1D5, M7047, dilution: 1:50; DAKO), or primary monoclonal mouse anti-progesterone receptor antibody (PgR636, M3569, dilution: 1:800; DAKO). The sections were then incubated with a secondary antibody (antirabbit, anti-mouse: DAKO) for $30 \mathrm{~min}$. Antibody binding was visualised using a 3,3'-diaminobenzidine solution (DAKO) for $10 \mathrm{~min}$. Finally, the tissues were counterstained by standard H\&E staining and mounted using a conventional mounting medium. All steps of IHC were performed at room temperature. An additional section was used as a case-specific negative control without incubation with the primary antibody. As a positive control, normal endometrial tissue was investigated. Evaluation of DLG1 IHC was performed semiquantitatively by light microscopy. The intensity of DLG1 staining was categorised as negative (score 0), weak (score 1), or strong (score 2). Weak cytoplasmic DLG1 expression was categorised as negative (score 0 ). Weak membranebound DLG1 expression was categorised as weak (score 1). Intense membrane-bound DLG1 expression was categorised as strong (score 2).

Statistical analysis. JMP 10 (SAS Institute, Tokyo, Japan) was used for statistical analysis. To analyse correlations between categorised variables, multi-field tables were calculated and interpreted using Pearson's $\chi^{2}$ test of independence. Risk ratios were estimated for clinical and pathological factors. Survival curves were estimated with Kaplan-Meier methods, and the respective curves were tested for significant differences by a log-rank test. Hazard ratios were estimated by Cox proportional hazards model. The level of statistical significance was set at $P<0.05$.

Cell culture. Seven endometrial cancer cell lines (KLE, Hec50B, Hec1B, HHUA, Hec108, and Hec151) and immortal human keratinocyte HaCaT cells were cultured in DMEM (Gibco, Grand Island, NY, USA) containing 10\% fetal bovine serum (Gibco). All cells were maintained at $37^{\circ} \mathrm{C}$ in $5 \% \mathrm{CO}_{2}$. Stable DLG1 knockdown cells were established in the KLE, Hec1B, and Hec59 cell lines.

Western blotting. Protein extracts were made in an NP-40 lysis buffer containing $100 \mathrm{~mm}$ Tris (pH 8.0), $100 \mathrm{~mm} \mathrm{NaCl}$, and $1 \%$ NP-40. Protein concentration was determined using a standard Bradford assay. Equal amounts of proteins were fractionated by SDS-PAGE and electrophoretically transferred onto a polyvinylidene difluoride membrane (Millipore, Bedford, MA, USA). An anti-DLG1 antibody (2D11, sc-9961; Santa Cruz) was used in a dilution of $1: 100$ to detect the expression of DLG1. For an internal control, the housekeeping enzyme glyceraldehyde 3-phosphate dehydrogenase (GAPDH) was selected. An anti-GAPDH antibody (ABS16; Millipore, Temecula, CA, USA) was used in a dilution of 
1:50 000. An ECL anti-rabbit horseradish peroxidase-conjugated secondary antibody (GE Healthcare, Little Chalfont, UK) was used in a dilution of $1: 10000$. The ECL Prime Western Blotting Detection Reagent (GE Healthcare) was used to detect hDlg and GAPDH expression.

Knockdown of $\boldsymbol{h D l g}$ with siRNA. We transfected nontargeting control small interfering RNA (siRNA) duplexes (sc-37007; Santa Cruz) and a specific duplex siRNA against $h D l g$ (sc-36452; Santa Cruz) into Hec108 cells using Lipofectamine RNAi MAX Reagent (Invitrogen) according to the manufacturer's instructions.

Establishment of stable DLG1 knockdown endometrial cancer cell lines. We transfected $2 \mu \mathrm{g}$ small hairpin RNA (shRNA) plasmid DNA (SAP 97 shRNA Plasmid (h), sc-36452-SH; Santa Cruz Biotechnology, Santa Cruz, CA, USA) into three endometrial cancer cell lines (KLE, Hec1B, and Hec59) cultured in $6 \mathrm{~cm}$ dishes using shRNA Plasmid Transfection Reagent (sc-108061; Santa Cruz Biotechnology) and shRNA Plasmid Transfection Medium (sc-108062; Santa Cruz Biotechnology). After $24 \mathrm{~h}$, the cells were incubated for $\sim 2$ weeks in medium containing puromycin $\left(2.5 \mu \mathrm{g} \mathrm{ml}^{-1}\right)$. We also established stable control shRNA expression clones in these cell lines. The drug-resistant clones were further incubated in the medium with puromycin and tested for the knockdown effect by western blotting using the anti-DLG1 antibody.

Wound healing assay. The migration of cells was evaluated using a wound healing assay. Linear wounds were created by scraping confluent cell monolayers with a pipette tip. The migration of cells into the wound area was documented and evaluated after 12 and $24 \mathrm{~h}$. We quantitated the wound healing assays in at least four independent experiments. The mean and s.d. (error bar) of at least four independent experiments are shown. The wound healing assays were performed in Hec108 cells into which the siRNA against $d l g 1$ was transfected. They were also performed in the stable DLG1 knockdown KLE, Hec1B, and Hec59 cells.

Matrigel chamber assay for migration and invasion. Quantitative cell migration and invasion assays were performed using a 24-well Matrigel Invasion Chamber (BD Biosciences, San Jose, CA, USA) containing a cell culture insert consisting of a PET membrane with a pore size of $8 \mu \mathrm{m}$ and coated with a thin layer of Matrigel basal membrane matrix. We measured the number of cells after trypsinisation and diluted them to have an equal amount of cells in each assay. After incubation at $37^{\circ} \mathrm{C}$ for $24 \mathrm{~h}$, the filters were collected and the cells that had adhered to the lower surface were fixed, stained with a Diff-Quik Stain Kit (Sysmex, Kobe, Japan), and counted. We quantitated the invasion rate in at least four independent experiments. The mean and s.d. (error bar) of at least four independent experiments are shown. The invasion assays were performed in Hec108 cells into which the siRNA against dlg1 was transfected. They were also performed in the stable DLG1 knockdown KLE, Hec1B, and Hec59 cells.

Cell cycle assay by flow cytometry. The cell cycle distribution of KLE cells and two stable human DLG1 knockdown KLE clones was analysed by flow cytometry. The cell cycle distribution was also analysed in Hec1B cells and two stable human DLG1 knockdown Hec1B clones. The cells were washed with phosphate-buffered saline (PBS) and fixed with $70 \%$ ethanol overnight at $4{ }^{\circ} \mathrm{C}$. The cells were stained with a BrdU Flow Kit (BD Biosciences), and cell cycle distribution was analysed by flow cytometry (FACSCanto II; BD Biosciences).

Soft agar colony formation assay. The endometrial cancer KLE cells and two stable DLG1 knockdown cells (clones 1 and 5) were incubated at $37^{\circ} \mathrm{C}$ and $5 \% \mathrm{CO}_{2}$ in semisolid agar medium using a CytoSelect Cell Transformation Assay (Cell Biolabs, San Diego, CA, USA). The cells were incubated for 8 days before being solubilised, lysed, and detected with CyQUANT GR Dye in a fluorescence plate reader using a $485 / 520 \mathrm{~nm}$ filter set. The transformed cell number was quantitated using relative fluorescence units (RFUs). The mean and s.d. (error bar) of the anchorage-independent growth ability of at least three independent experiments are shown. The anchorage-independent growth was also analysed in Hec1B cells and two stable DLG1 knockdown clones.

\section{RESULTS}

The IHC analysis showed that DLG1 was localised at the basolateral membrane in normal endometrial tissues in the proliferative and secretory phases (Figure $1 \mathrm{~A}$ and B). Human DLG1 expression was more abundant in the lower part of the basolateral membrane in endometrial tissues in the secretory phase (Figure 1B). Next, we analysed DLG1 expression and localisation in 160 cases of endometrial cancer. Strong membrane-bound expression of DLG1 (DLG1 score 2) was observed in 37 cases (23.1\%) (Figure 1C). Weak membrane-bound hDlg expression (DLG1 score 1) was observed in 46 cases (28.8\%) (Figure 1D). Weak cytoplasmic hDlg expression (DLG1 score 0) was observed in 77 cases $(48.1 \%)$ (Figure $1 \mathrm{E})$.

We evaluated whether loss of DLG1 has a link with the prognosis of patients with endometrial cancer. Patients with loss of DLG1 expression (DLG1 score 0) showed poorer overall survival than those with strong or weak membrane-bound expression of DLG1 (DLG1 score 2 or 1$)(P=0.0018$, log-rank test) (Figure 2). Patients with loss of DLG1 expression also showed poorer progression-free survival than those with DLG1 expression (DLG1 score 2 or 1), but the difference did not reach statistical significance $(P=0.0752)$.

The ratio of patients with advanced clinical stage (stages 3 and 4) to those with early clinical stage was $24.3 \%, 19.6 \%$, and $14.3 \%$ in patients with DLG1 scores 0,1 , and 2, respectively. The proportion of patients with advanced clinical stage to those with early clinical
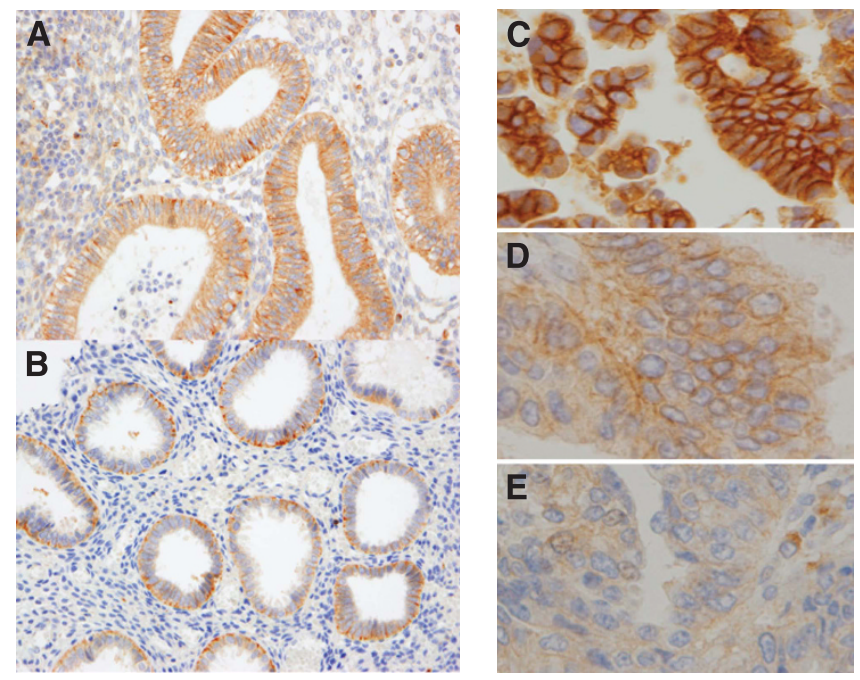

Figure 1. Immunohistochemical analysis of DLG1 expression in normal and malignant endometrial tissues. (A) Expression of DLG1 in normal endometrium in the proliferative phase. (B) Expression of DLG1 in normal endometrium in the secretory phase. Human DLG1 expression was more abundant in the lower part of the basolateral membrane in endometrial tissues. (C) Strong membrane-bound expression of DLG1 in endometrial cancer (hDlg score 2). (D) Membrane-bound weak DLG1 expression in endometrial cancer (hDlg score 1). (E) Weak cytoplasmic DLG1 expression in endometrial cancer (hDlg score 0). 
stage increased with loss of DLG1 expression (Table 1, not significant). Lymph node involvement was observed in $42.3 \%$, $17.9 \%$, and $15.8 \%$ of patients with DLG1 scores 0,1 , and 2, respectively (Table 1). The nodal metastasis rate increased with loss of hDlg expression $(P=0.0138)$. The DLG1 score was not

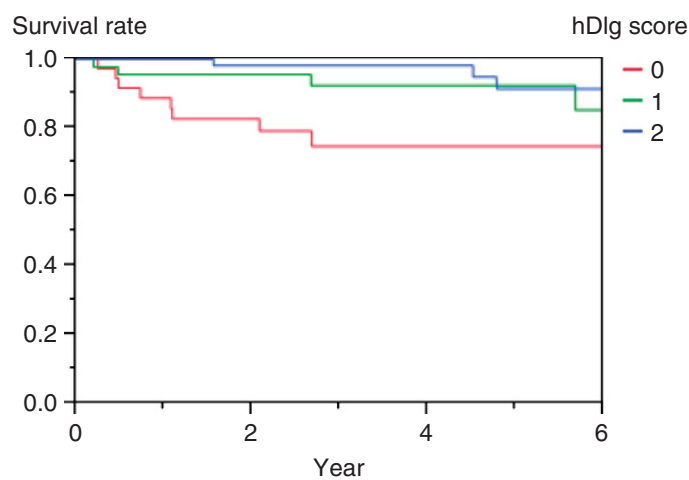

Figure 2. Overall survival of patients with endometrial cancer according to DLG1 expression score. Patients with loss of DLG1 expression (DLG1 score 0) showed poorer overall survival than those with strong or weak membrane-bound expression of DLG1 (DLG1 score 2 or 1$)(P=0.0018$, log-rank test). correlated with distant metastasis, lymph and vascular vessel involvement, or age at onset (Table 1). Deep myometrial invasion (over $50 \%$ of the myometrium) was observed in $51.4 \%, 43.5 \%$, and $28.6 \%$ of patients with DLG1 scores 0 , 1, and 2, respectively (Table 1). Loss of DLG1 showed a significant correlation with deep myometrial invasion $(P=0.0134)$. The rate of high-grade histology was $43.2 \%, 19.6 \%$, and $9.1 \%$ in patients with DLG1 scores 0,1 , and 2 , respectively (Table 1 ). The proportion of patients with highgrade histology showed an inverse correlation with the hDlg score $(P<0.0001)$. Loss of DLG1 expression showed a significant correlation with negative oestrogen and progesterone receptor expression (Table $1, P<0.0001$ ). Mutation of p53 has been shown to be a biomarker for type 2 endometrial cancer (Inoue et al, 1994). We investigated the relationship between loss of DLG1 and p53 mutation; however, there was no significant relationship between them (Table 1). Next, we analysed E-cadherin (CDH1) expression and compared it with loss of DLG1; however, there was no relationship between the loss of $\mathrm{CDH} 1$ expression and loss of DLG1 (Table 1). These results support the possibility that loss of DLG1 is a novel molecular marker of type 2 signatures in endometrial cancer.

Next, we evaluated DLG1 expression by quantitative RT-PCR in endometrial cancer tissues. $\beta$-Actin expression was evaluated as an internal control. The quantitative expression intensity of DLG1 against $\beta$-actin showed a positive correlation with the IHC DLG1 score (Figure 3).

Table 1. Relationship between human Discs-large (hDlg) expression and clinicopathological characters in endometrial cancer

\begin{tabular}{|c|c|c|c|c|c|}
\hline Variables & Total & hDlg score 0 & hDlg score 1 & hDlg score 2 & $P$-value \\
\hline $\begin{array}{l}\text { Overall survival } \\
\text { Progression-free survival }\end{array}$ & & & & & $\begin{array}{l}0.0018 \\
0.6281\end{array}$ \\
\hline \multicolumn{6}{|l|}{ Age (years) } \\
\hline $\begin{array}{l}<50 \\
\geqslant 50\end{array}$ & $\begin{array}{r}47 \\
113\end{array}$ & $\begin{array}{l}21.3 \%(10) \\
23.9 \%(27)\end{array}$ & $\begin{array}{l}27.7 \%(13) \\
29.2 \%(33)\end{array}$ & $\begin{array}{l}51.1 \%(24) \\
46.9 \%(53)\end{array}$ & 0.6281 \\
\hline \multicolumn{6}{|l|}{ Stage } \\
\hline $\begin{array}{l}\text { pT1-2 } \\
\text { pT3-4 }\end{array}$ & $\begin{array}{r}131 \\
29\end{array}$ & $\begin{array}{l}21.4 \%(28) \\
31.0 \%(9)\end{array}$ & $\begin{array}{l}28.2 \%(37) \\
31.0 \%(9)\end{array}$ & $\begin{array}{l}50.4 \%(66) \\
37.9 \%(11)\end{array}$ & 0.181 \\
\hline \multicolumn{6}{|c|}{ Lymph nodal metastasis } \\
\hline $\begin{array}{l}\text { pN0 } \\
\text { pN1 }\end{array}$ & $\begin{array}{l}95 \\
27\end{array}$ & $\begin{array}{l}15.8 \%(15) \\
40.7 \%(11)\end{array}$ & $\begin{array}{l}33.7 \%(32) \\
25.9 \%(7)\end{array}$ & $\begin{array}{l}50.5 \%(48) \\
33.3 \%(9)\end{array}$ & 0.0138 \\
\hline \multicolumn{6}{|l|}{ Distant metastasis } \\
\hline $\begin{array}{l}\text { M0 } \\
\text { M1 }\end{array}$ & $\begin{array}{r}148 \\
12\end{array}$ & $\begin{array}{l}22.3 \%(33) \\
33.3 \%(4)\end{array}$ & $\begin{array}{l}29.7 \%(44) \\
16.7 \%(2)\end{array}$ & $\begin{array}{l}48.0 \%(71) \\
50.0 \%(6)\end{array}$ & 0.7097 \\
\hline \multicolumn{6}{|l|}{ Vessel involvement } \\
\hline $\begin{array}{l}- \\
+\end{array}$ & $\begin{array}{r}101 \\
59\end{array}$ & $\begin{array}{l}20.8 \%(21) \\
27.1 \%(16)\end{array}$ & $\begin{array}{l}25.7 \%(26) \\
33.9 \%(20)\end{array}$ & $\begin{array}{l}53.5 \%(54) \\
39 / 0 \%(23)\end{array}$ & 0.1152 \\
\hline \multicolumn{6}{|l|}{ Myometrial invasion } \\
\hline $\begin{array}{l}<1 / 2 \\
\geqslant 1 / 2\end{array}$ & $\begin{array}{l}99 \\
61\end{array}$ & $\begin{array}{l}18.2 \%(18) \\
31.1 \%(19)\end{array}$ & $\begin{array}{l}26.3 \%(26) \\
32.8 \%(20)\end{array}$ & $\begin{array}{l}55.6 \%(55) \\
36.1 \%(22)\end{array}$ & 0.0134 \\
\hline \multicolumn{6}{|l|}{ Histological grade } \\
\hline $\begin{array}{l}\text { Low grade } \\
\text { High grade }\end{array}$ & $\begin{array}{r}128 \\
32\end{array}$ & $\begin{array}{l}16.4 \%(21) \\
50.0 \%(16)\end{array}$ & $\begin{array}{l}28.9 \%(37) \\
28.1 \%(9)\end{array}$ & $\begin{array}{l}54.7 \%(70) \\
21.9 \%(7)\end{array}$ & $<0.0001$ \\
\hline \multicolumn{6}{|l|}{ Oestrogen receptor } \\
\hline $\begin{array}{l}- \\
+\end{array}$ & $\begin{array}{r}26 \\
134\end{array}$ & $\begin{array}{l}76.9 \%(20) \\
12.7 \%(17)\end{array}$ & $\begin{array}{l}15.4 \%(4) \\
31.3 \%(42)\end{array}$ & $\begin{array}{c}7.7 \%(2) \\
56.0 \%(75)\end{array}$ & $<0.0001$ \\
\hline \multicolumn{6}{|l|}{ Progesterone receptor } \\
\hline $\begin{array}{l}- \\
+\end{array}$ & $\begin{array}{r}35 \\
125\end{array}$ & $\begin{array}{l}57.1 \%(20) \\
13.6 \%(17)\end{array}$ & $\begin{array}{l}25.7 \%(9) \\
29.6 \%(37)\end{array}$ & $\begin{array}{l}17.1 \%(6) \\
56.8 \%(71)\end{array}$ & $<0.0001$ \\
\hline \multicolumn{6}{|l|}{ p53 } \\
\hline $\begin{array}{l}\text { Mutation } \\
\text { Wild }\end{array}$ & $\begin{array}{r}26 \\
130\end{array}$ & $\begin{array}{l}26.9 \%(7) \\
23.1 \%(30)\end{array}$ & $\begin{array}{l}34.6 \%(9) \\
28.5 \%(37)\end{array}$ & $\begin{array}{l}38.5 \%(10) \\
48.5 \%(63)\end{array}$ & 0.4247 \\
\hline \multicolumn{6}{|l|}{ E-cadherin } \\
\hline $\begin{array}{l}\text { Down regulation } \\
\text { Normal expression }\end{array}$ & $\begin{array}{r}38 \\
114\end{array}$ & $\begin{array}{l}34.2 \%(13) \\
20.2 \%(23)\end{array}$ & $\begin{array}{l}21.1 \%(8) \\
33.3 \%(38)\end{array}$ & $\begin{array}{l}44.7 \%(17) \\
46.5 \%(53)\end{array}$ & 0.2948 \\
\hline
\end{tabular}


We established stable human DLG1 knockdown clones for three endometrial cancer cell lines KLE, Hec1B, and Hec59 (KLE clones 1 and 5, Figure 4A). We analysed whether DLG1 was involved in tumour migration by using wound healing and invasion assays in these cells. Representative data of the wound healing assay are shown in Figure 4B.

The stable human DLG1 knockdown KLE cells (clone 1) showed increased migration compared with the control shRNAtransfected KLE cells (Figure 4C). Transfection of siRNA against $h D l g$ increased migration in Hec108 cells (Figure 4C). In contrast, the stable human DLG1 knockdown Hec1B, Hec59 clone, and KLE clone 5 did not show increased migration.

Furthermore, we carried out migration and invasion assays using the Boyden chamber system. Knockdown of $h D l g$ expression

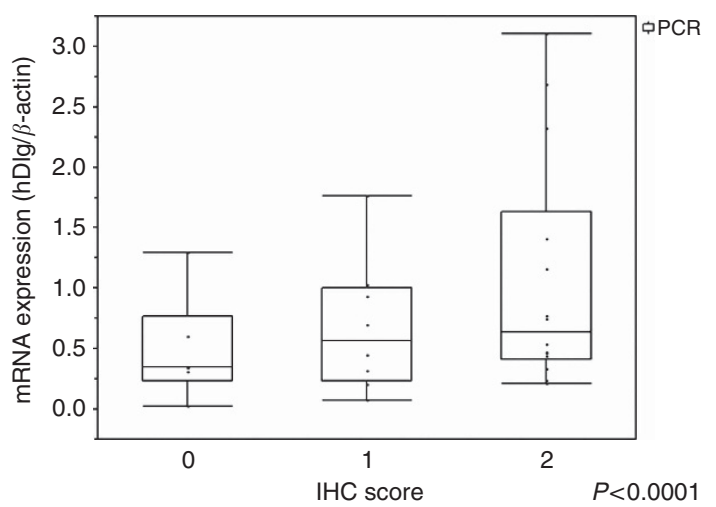

Figure 3. Correlation between DLG1 expression intensity analysed by quantitative RT-PCR and DLG1 IHC score. The quantitative expression intensity of DLG1 against $\beta$-actin showed a positive correlation with the IHC DLG1 score. by siRNA significantly enhanced the migration and invasion ability of Hec108 cells (Figure 4D, $P=0.0034$ ). The stable human DLG1 knockdown KLE clones showed increased invasion ability compared with the control shRNA-transfected KLE cells (Figure 4D).

We investigated whether loss of DLG1 is involved in anchorageindependent growth. Higher colony-forming ability in soft agar medium was observed in the stable DLG1 knockdown KLE clone 1 cells than in the control shRNA-transfected KLE cells $(P=0.046$, Figure 5A). In contrast, the stable DLG1 knockdown Hec clone 7 cells showed lower colony-forming ability compared with the control shRNA-transfected Hec1B cells $(P=0.0174$, Figure $5 \mathrm{~A})$.

Next, we analysed whether human DLG1 has a role in cell cycle regulation. We analysed cell cycle distribution in the control shRNA-transfected cells and human DLG1 knockdown clones. In the DLG1 knockdown KLE clones, a slight increase of cells in the S phase was observed, but this trend was not observed in the Hec1B knockdown clones (Figure 5C, statistically not significant).

\section{DISCUSSION}

The relationship between the loss of epithelial polarity and malignant tumour development has long been known, but whether it is merely a coincidence or whether loss of tissue architecture might contribute to tumourigenesis remains a compelling question (Bilder, 2004). Drosophila tumour suppressors are divided into two groups: 'hyperplastic' and 'neoplastic' tumour suppressors. In a hyperplastic tumour, imaginal discs contain increased numbers of cells, but the cells remain arranged in an epithelial monolayer, despite extensive overproliferation (Bilder, 2004). Hyperplastic tumours are caused by inactivating mutations in genes involved in cell growth regulation and organ size control (Bilder, 2004). Neoplastic tumours, in which the tumour suppressor genes $l g l, d l g$, or scrib are mutated, show disruption of the organised epithelial

B KLE

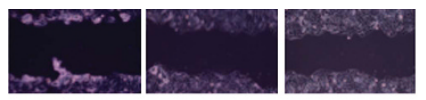

KLE control sh
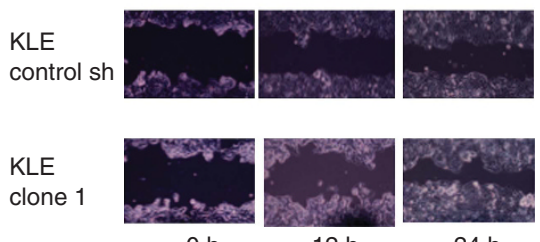

D

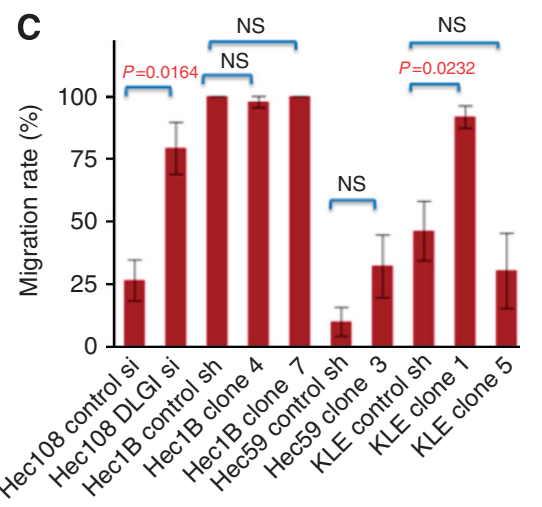

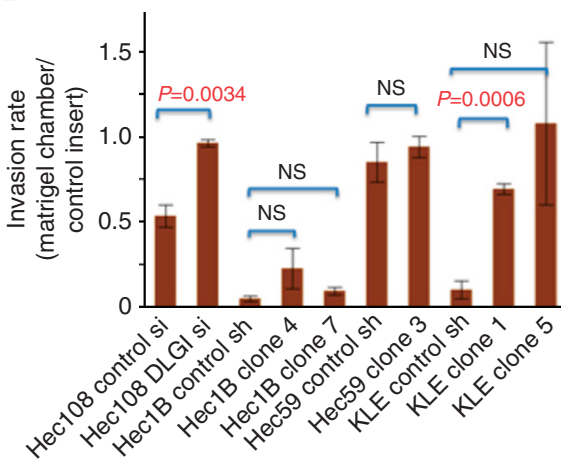

Figure 4. Knockdown of DLG1 expression by siRNA and shRNA significantly enhanced the migration and invasion ability of endometrial cancer cells. (A) Western blotting analysis of DLG1 and GAPDH expression in the stable DLG1 knockdown KLE clones. The silencing of DLG1 was observed in the clones 1 and 5. (B) Representative data of the wound healing assay. (C) The wound healing assay in Hec108, Hec1B, Hec59, and KLE cells. (D) Invasion assay using the Boyden chamber system in Hec108, Hec1B, Hec59, and KLE cells. 


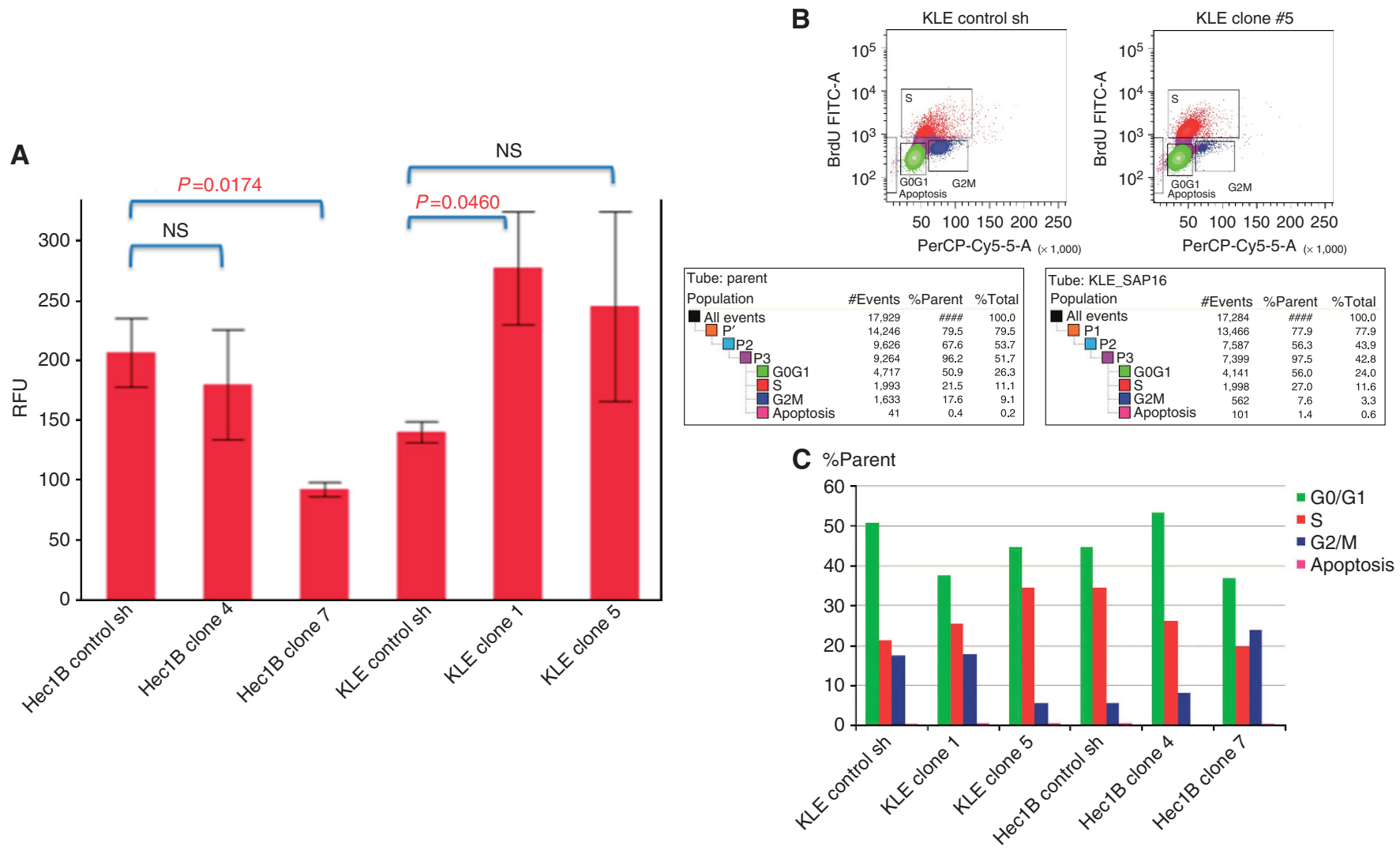

Figure 5. Anchorage-independent growth and cell cycle distribution analysis. (A) Anchorage-independent growth assay in Hec1B and KLE cells. (B) Representative data of cell cycle distribution analysed by flow cytometry of KLE cells and stable human DLG1 knockdown KLE cells.

(C) Quantitative analysis of cell cycle distribution in the human DLG1 knockdown endometrial cancer cells.

monolayer. Neoplastic tumour cells are rounded rather than cuboidal and pile up atop one another as they overproliferate. The imaginal discs of neoplastic tumour suppressor gene mutant larvae are a spherical mass and much larger than their wild-type counterpart (Bilder, 2004).

Several lines of evidence support the possibility that DLG1 is involved in mammalian tumourigenesis (Humbert et al, 2008). Human papillomavirus (HPV) is a causal factor of cervical cancer (zur Hausen, 2002). Ubiquitin-mediated degradation of the tumour suppressor proteins $\mathrm{p} 53$ and $\mathrm{pRb}$ has been shown to be a central mechanism underlying cervical carcinogenesis (Howley, 2006). High-risk, but not low-risk, HPV E6 oncoprotein binds to DLG1 and induces its ubiquitin-mediated degradation (Kiyono et al, 1997; Gardiol et al, 1999; Matsumoto et al, 2006). Loss of the membrane-bound expression of DLG1 has been reported during cervical cancer development from normal cervical epithelial tissue to a premalignant lesion (Watson et al, 2002). Loss of DLG1 expression was reported during colon cancer development (Gardiol et al, 2006); it was observed especially in colon cancer with undifferentiated histology (Gardiol et al, 2006). Somatic missense mutations were found in PDZ domain 2 of hDlg in mammary ductal carcinoma (Fuja et al, 2004). Human DLG1 binds directly to adenomatous polyposis coli (APC), which is an important Wnt pathway component, through PDZ domain 2. Somatic mutation in this region might affect the Wnt pathway by interfering with its interaction with APC during breast cancer development. It is possible that loss of DLG1 found in endometrial cancer might be because of somatic mutation. Sequencing analysis of DLG1 in endometrial cancer tissues is undergoing in our laboratory. A recent study showed a high frequency of $\beta$-catenin mutation in endometrial cancer (Cancer Genome Atlas Research Network, et al, 2013). There is a possibility that loss of $\beta$-catenin, which is an important Wnt pathway component, might affect the stability of DLG1 expression.
Here, we provide novel evidence that support a role for DLG1 in mammalian tumour development. Our study showed that loss of DLG1 is linked with deep myometrial invasion, nodal metastasis, and poor prognosis in endometrial cancer. Loss of DLG1 also demonstrated a negative relationship with the presence of oestrogen and progesterone receptors. Our data suggested that endometrial cancer with loss of DLG1 might share type 2 characteristics. CDH1 (E-cadherin) resides at the adherens junction and is a critical molecule that is involved in tissue polarity. Loss of $\mathrm{CDH} 1$ expression is reportedly associated with the type 2 characteristics of endometrial cancer (Carico et al, 2010). Our study showed that loss of DLG1 is an independent phenomenon to the loss of CDH1. The overexpression of p53 due to mutation is a biomarker for poor prognosis and type 2 characteristics of endometrial cancer (Inoue et al, 1994; Ito et al, 1994). We analysed the relationship between loss of DLG1 and p53 overexpression in our cases. However, there was no relationship between these molecular markers (Table 1), suggesting that loss of DLG1 is a novel molecular marker for the invasiveness and metastatic behaviour of endometrial cancer. Our in vitro data support the possibility that loss of DLG1 is involved in accelerated migration and invasion of endometrial cancer. Higher lymph nodal metastasis and deep myometrial invasion found in patients with loss of DLG1 might support this possibility.

Previously, we identified the human homologue of Drosophila Scribble, human SCRIB, as a target for ubiquitin-mediated degradation depending on HPV E6 oncoprotein and E3 protein-ubiquitin ligase, E6AP (Nakagawa and Huibregtse, 2000). Progressive loss of the expression of DLG1 and human SCRIB is observed in human tumours and their precursor lesions (Watson et al, 2002; Nakagawa et al, 2004). Transgenic mice homozygous for mutations of Dlg or Scrib exhibit craniofacial dysmorphogenesis or neural tube defects (Murdoch et al, 2003). These data 
support the possibility that DLG1 and human SCRIB share a role in the control of tissue architecture and the suppression of tumourigenesis. The decreased expression and changed localisation of human SCRIB have been shown to be associated with advanced clinical stage, histological differentiation, and nodal metastasis in endometrial cancer (Ouyang et al, 2010). Our previous study showed that loss of Hugl-1 expression was associated with nodal metastasis and poor prognosis (Tsuruga et al, 2007). These studies suggest the possibility that the human homologues of Drosophila neoplastic tumour suppressors share a role in the suppression of development and progression of endometrial cancer. These data shed light on the possibility that three human homologues of Drosophila neoplastic tumour suppressors are involved in the establishment of tissue architecture in concert, similar to their Drosophila homologues. It will be of interest to investigate whether loss of these tumour suppressors overlaps or is mutually exclusive. Analysis of human SCRIB and Hugl-1 in these patients with endometrial cancer is undergoing in our laboratory.

In conclusion, our study revealed that loss of DLG1 is a novel molecular marker for poor prognosis in endometrial cancer.

\section{ACKNOWLEDGEMENTS}

We thank M Watanebe for his scientific and technical support. This study was supported by a Grant-in-Aid (Number $15 \mathrm{~cm} 0106061 \mathrm{~h} 0005)$ for Scientific Research from the Ministry of Education, Science, and Culture, Japan.

\section{CONFLICT OF INTEREST}

The authors declare no conflict of interest.

\section{REFERENCES}

Bansal N, Yendluri V, Wenham RM (2009) The molecular biology of endometrial cancers and the implications for pathogenesis, classification, and targeted therapies. Cancer Control 16(1): 8-13.

Barrena Medel NI, Herzog TJ, Deutsch I, Burke WM, Sun X, Lewin SN, Wright JD (2011) Comparison of the prognostic significance of uterine factors and nodal status for endometrial cancer. Am J Obstet Gynecol 204(3): 248 e1-7.

Bilder D (2004) Epithelial polarity and proliferation control: links from the Drosophila neoplastic tumor suppressors. Genes Dev 18(16): 1909-1925.

Bokhman JV (1983) Two pathogenetic types of endometrial carcinoma. Gynecol Oncol 15(1): 10-17.

Cancer Genome Atlas Research NetworkKandoth C, Schultz N, Cherniack AD, Akbani R, Liu Y, Shen H, Robertson AG, Pashtan I, Shen R, Benz CC, Yau C, Laird PW, Ding L, Zhang W, Mills GB, Kucherlapati R, Mardis ER, Levine DA (2013) Integrated genomic characterization of endometrial carcinoma. Nature 497(7447): 67-73.

Carico E, Atlante M, Giarnieri E, Raffa S, Bucci B, Giovagnoli MR, Vecchione A (2010) E-cadherin and alpha-catenin expression in normal, hyperplastic and neoplastic endometrium. Anticancer Res 30(12): 4993-4997.

Doll A, Abal M, Rigau M, Monge M, Gonzalez M, Demajo S, Colas E, Llaurado M, Alazzouzi H, Planaguma J, Lohmann MA, Garcia J, Castellvi S, Ramon y Cajal J, Gil-Moreno A, Xercavins J, Alameda F, Reventos J (2008) Novel molecular profiles of endometrial cancer-new light through old windows. J Steroid Biochem Mol Biol 108(3-5): 221-229.

Ferlay J, Shin HR, Bray F, Forman D, Mathers C, Parkin DM (2010) Estimates of worldwide burden of cancer in 2008: GLOBOCAN 2008. Int J Cancer 127(12): 2893-2917.

Fuja TJ, Lin F, Osann KE, Bryant PJ (2004) Somatic mutations and altered expression of the candidate tumor suppressors CSNK1 epsilon, DLG1, and EDD/hHYD in mammary ductal carcinoma. Cancer Res 64(3): 942-951.
Gardiol D, Kuhne C, Glaunsinger B, Lee SS, Javier R, Banks L (1999) Oncogenic human papillomavirus E6 proteins target the discs large tumour suppressor for proteasome-mediated degradation. Oncogene 18(40): 5487-5496.

Gardiol D, Zacchi A, Petrera F, Stanta G, Banks L (2006) Human discs large and scrib are localized at the same regions in colon mucosa and changes in their expression patterns are correlated with loss of tissue architecture during malignant progression. Int J Cancer 119(6): 1285-1290.

Hanahan D, Weinberg RA (2011) Hallmarks of cancer: the next generation. Cell 144(5): 646-674.

Howley PM (2006) Warts, cancer and ubiquitylation: lessons from the papillomaviruses. Trans Am Clin Climatol Assoc 117: 113-126 discussion 126-7.

Humbert PO, Grzeschik NA, Brumby AM, Galea R, Elsum I, Richardson HE (2008) Control of tumourigenesis by the Scribble/Dlg/Lgl polarity module. Oncogene 27(55): 6888-6907.

Inoue $\mathrm{M}$, Okayama $\mathrm{A}$, Fujita $\mathrm{M}$, Enomoto T, Sakata $\mathrm{M}$, Tanizawa $\mathrm{O}$, Ueshima $\mathrm{H}$ (1994) Clinicopathological characteristics of p53 overexpression in endometrial cancers. Int J Cancer 58(1): 14-19.

Ito K, Watanabe K, Nasim S, Sasano H, Sato S, Yajima A, Silverberg SG, Garrett CT (1994) Prognostic significance of p53 overexpression in endometrial cancer. Cancer Res 54(17): 4667-4670.

Ivanova S, Gregorc U, Vidergar N, Javier R, Bredt DS, Vandenabeele P, Pardo J, Simon MM, Turk V, Banks L, Turk B (2011) MAGUKs, scaffolding proteins at cell junctions, are substrates of different proteases during apoptosis. Cell Death Dis 2: e116.

Jemal A, Siegel R, Xu J, Ward E (2010) Cancer statistics, 2010. CA Cancer J Clin 60(5): 277-300.

Kiyono T, Hiraiwa A, Fujita M, Hayashi Y, Akiyama T, Ishibashi M (1997) Binding of high-risk human papillomavirus $\mathrm{E} 6$ oncoproteins to the human homologue of the Drosophila discs large tumor suppressor protein. Proc Natl Acad Sci USA 94(21): 11612-11616.

Matsumoto Y, Nakagawa S, Yano T, Takizawa S, Nagasaka K, Nakagawa K, Minaguchi T, Wada O, Ooishi H, Matsumoto K, Yasugi T, Kanda T, Huibregtse JM, Taketani Y (2006) Involvement of a cellular ubiquitin-protein ligase E6AP in the ubiquitin-mediated degradation of extensive substrates of high-risk human papillomavirus E6. J Med Virol 78(4): 501-507.

Mendivil A, Schuler KM, Gehrig PA (2009) Non-endometrioid adenocarcinoma of the uterine corpus: a review of selected histological subtypes. Cancer Control 16(1): 46-52.

Murdoch JN, Henderson DJ, Doudney K, Gaston-Massuet C, Phillips HM, Paternotte C, Arkell R, Stanier P, Copp AJ (2003) Disruption of scribble (Scrb1) causes severe neural tube defects in the circletail mouse. Hum Mol Genet 12(2): 87-98.

Nakagawa S, Huibregtse JM (2000) Human scribble (Vartul) is targeted for ubiquitin-mediated degradation by the high-risk papillomavirus E6 proteins and the E6AP ubiquitin-protein ligase. Mol Cell Biol 20(21): $8244-8253$

Nakagawa S, Yano T, Nakagawa K, Takizawa S, Suzuki Y, Yasugi T, Huibregtse JM, Taketani Y (2004) Analysis of the expression and localisation of a LAP protein, human scribble, in the normal and neoplastic epithelium of uterine cervix. Br J Cancer 90(1): 194-199.

Ouyang Z, Zhan W, Dan L (2010) hScrib, a human homolog of Drosophila neoplastic tumor suppressor, is involved in the progress of endometrial cancer. Oncol Res 18(11-12): 593-599.

Tsuruga T, Nakagawa S, Watanabe M, Takizawa S, Matsumoto Y, Nagasaka K, Sone K, Hiraike H, Miyamoto Y, Hiraike O, Minaguchi T, Oda K, Yasugi T, Yano T, Taketani Y (2007) Loss of Hugl-1 expression associates with lymph node metastasis in endometrial cancer. Oncol Res 16(9): 431-435.

Watson RA, Rollason TP, Reynolds GM, Murray PG, Banks L, Roberts S (2002) Changes in expression of the human homologue of the Drosophila discs large tumour suppressor protein in high-grade premalignant cervical neoplasias. Carcinogenesis 23(11): 1791-1796.

zur Hausen H (2002) Papillomaviruses and cancer: from basic studies to clinical application. Nat Rev Cancer 2(5): 342-350.

This work is published under the standard license to publish agreement. After 12 months the work will become freely available and the license terms will switch to a Creative Commons AttributionNonCommercial-Share Alike 4.0 Unported License. 